\title{
Las especies en peligro de extinción y los mecanismos para la recuperación y conservación de la biodiversidad: un estudio sobre la viabilidad de los mecanismos y las trabas burocráticas
}

\section{Endangered Species and Mechanisms for the Recovery and Conservation of Biodiversity: A Study on the Viability of Mechanisms and Bureaucratic Obstacles}

Marcelo López Alfonsín* https://orcid.org/0000-0002-2110-3354

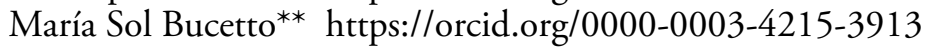

http://dx.doi.org/10.21503/lex.v17i23.1680

* Juez de la Ciudad Autónoma de Buenos Aires (Argentina). Doctor en Derecho, Área Derecho Constitucional (Universidad de Buenos Aires, Argentina). Magíster en Derecho Ambiental (Universidad de Lomas de Zamora, Argentina).

Correo electrónico: mlalfonsin@jusbaires.gov.ar

** Abogada de la Universidad de Buenos Aires, candidata a magíster en Derechos Humanos, Universidad Nacional de La Plata. Docente de "Los Derechos Humanos en la Constitución Nacional”, Universidad de Buenos Aires.

Correo electrónico: solbucetto@yahoo.com.ar

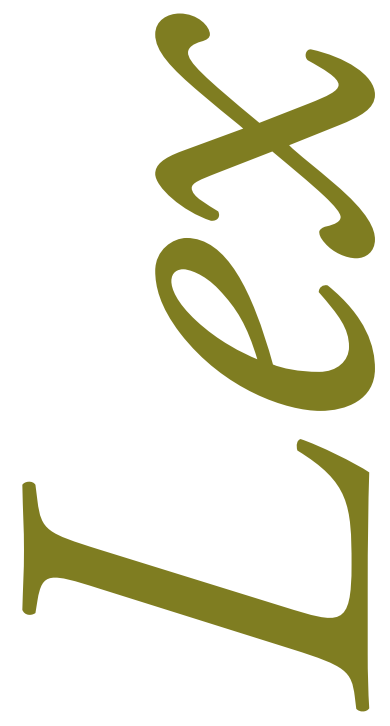




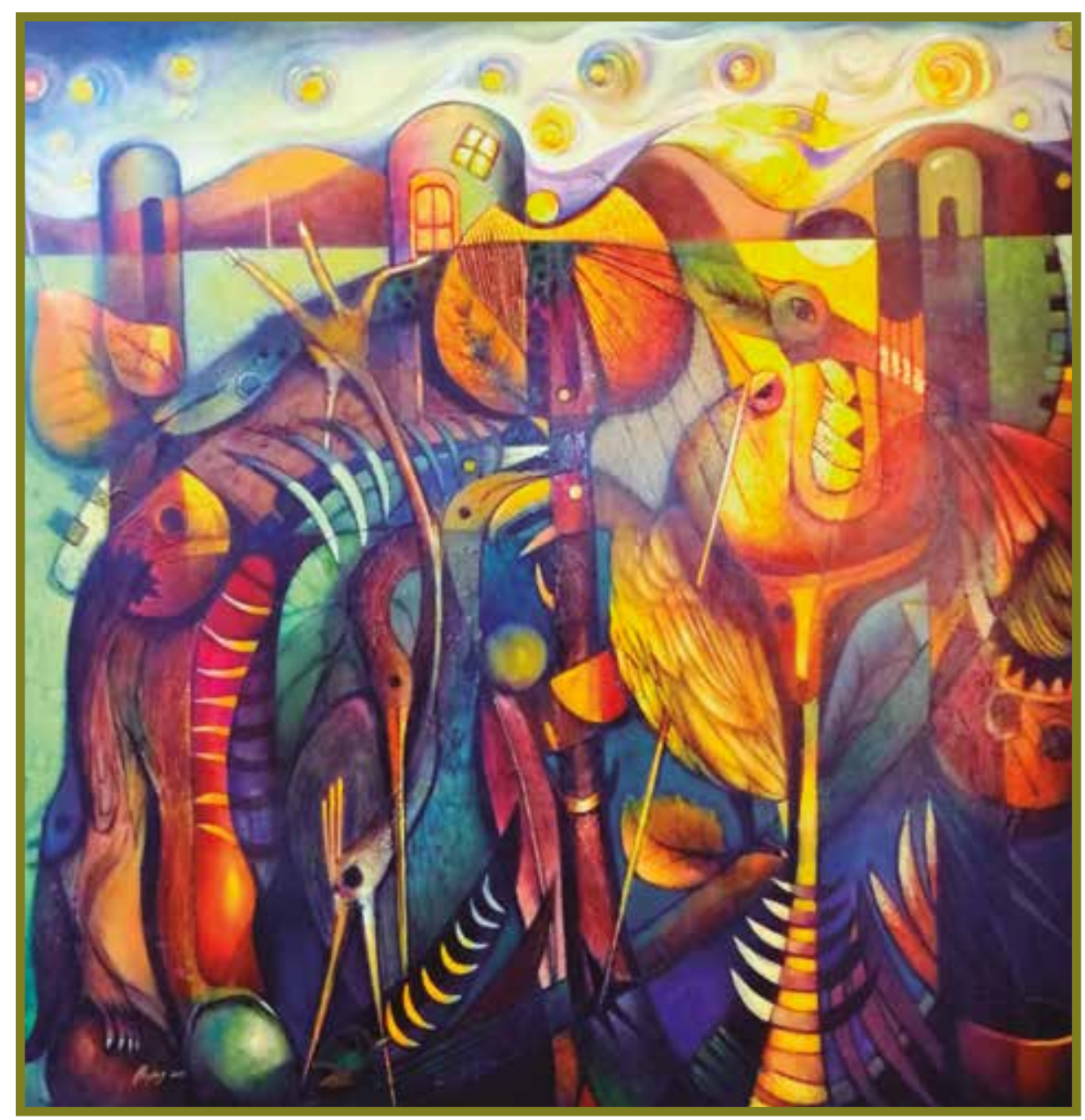

Contaminación. Óleo 100 x $100 \mathrm{~cm}$.

Agustín Aquino Mejías (pintor peruano). 


\section{RESUMEN}

La biodiversidad mundial se ha visto afectada por el fenómeno de la extinción de especies. La UICN utiliza categorías y criterios preestablecidos que tienen la intención de ser un sistema de fácil comprensión para clasificar especies en alto riesgo de extinción global. Para hacer frente a este dramático escenario, la mejor estrategia es la preservación del medio natural, pero cuando ello no es posible existen otras propuestas como lo dispuesto en la Ley de Especies en Peligro de Extinción (ESA) de Estados Unidos y los mecanismos de incentivo y los centros de reproducción en cautividad y las biotecnologías reproductivas. Todos estos procesos están sujetos a tediosos escollos burocráticos que dificultan el éxito de los programas. Al analizar el caso del huemul, en tanto monumento natural argentino, consideramos que el futuro de la recuperación y conservación se encuentra en los centros de reproducción en cautiverio.

Palabras clave: especies en peligro de extinción, Unión Internacional para la Conservación de la Naturaleza (UICN), Convención sobre el Comercio Internacional de Especies Amenazadas de Fauna y Flora Silvestres (CITES), áreas protegidas, Ley de Especies en Peligro de Extinción (ESA) de Estados Unidos, centros de reproducción en cautividad y biotecnologias reproductivas, lobo mexicano, huemul.

\section{ABSTRACT}

The world's biodiversity has been affected by the phenomenon of extinction of the species. IUCN uses pre-established categories and criteria that are intended to be an easily understood system for classifying species at high risk of global extinction. To face this dramatic scenario, the best strategy is the preservation of the natural environment, but when this is not possible there are other proposals, such as the previsions of the Endangered Species Act (ESA) and mechanisms of Incentives and centers of reproduction in captivity and reproductive biotechnologies. All these processes are subject to the bureaucratic obstacles that hinder the success of the programs. When analyzing the case of the huemul, as an Argentinian natural monument, we believe that the future of recovery and conservation is found in centers of reproduction in captivity.

Key words: endangered species, International Union for the Conservation of Nature (IUCN), Convention on International Trade in Endangered Species of Wild Fauna and Flora (CITES), protected areas, Endangered Species Act (ESA), centers of reproduction in captivity and reproductive biotechnologies, mexican wolf, huemul. 


\section{INTRODUCCIÓN}

El ambiente puede ser definido como:

(...) aquel sistema global constituido por elementos naturales, artificiales de naturaleza, física, química o biológica, socioculturales y sus interacciones, en permanente modificación por la acción humana o natural, que rige y condiciona la existencia y el desarrollo de la vida en sus múltiples manifestaciones. ${ }^{1}$

A partir de este axioma, la ecología comenzó a relacionarse con las ciencias sociales, analizando los procesos por los cuales el hombre puede modificar el equilibrio de los ecosistemas, con las consiguientes repercusiones para el ambiente y el propio desarrollo de la vida humana.

En estas coordenadas debemos situarnos cuando hablamos de paradigma ambiental. Esta nueva concepción da lugar a la aparición de las obligaciones de protección de los bienes colectivos y a las restricciones sobre el pleno ejercicio de los derechos individuales, que sean susceptibles de afectar al ambiente. ${ }^{2}$

La biodiversidad mundial se ha visto afectada por el fenómeno de la extinción de especies. Las causas principales son la pérdida y degradación del hábitat, el avance de la frontera agropecuaria, la caza ilegal, el tráfico de especies o de productos derivados, la contaminación, el cambio climático, la introducción de especies exóticas y el sobrepastoreo.

Hoy en día, la tasa de extinción de animales es de diez a cien veces superior a la que existiría sin la intervención humana, y se acelerará en las próximas décadas. Es por eso que las iniciativas se multiplican para preservar la biodiversidad: creación de reservas naturales, estudio del funcionamiento de los ecosistemas, etc. ${ }^{3}$

R. Lorenzetti, La protección jurídica del ambiente, LL, 1997-E-1463.

M. López Alfonsín, Derecho ambiental (Buenos Aires: Astrea, 2012), 29.

3 J. Espunyes Nozières, Reintroducción de especies amenazadas. Problemáticas y recomendaciones (España: Universitat Autónoma de Barcelona, 2011/2012), https://ddd.uab.cat/pub/trerecpro/2011/85780/reiespame.pdf 
En el presente artículo haremos un repaso por la categorización de las especies en "vulnerables", "amenazadas" y "en peligro de extinción", a fin de comprender los criterios de clasificación y sus consecuencias en el plano legal. Para ello tomaremos los criterios universales de la Unión Internacional para la Conservación de la Naturaleza (UICN) y la Convención sobre el Comercio Internacional de Especies Amenazadas de Fauna y Flora Silvestres (CITES).

Con el objetivo de comparar las categorías que se utilizan a nivel mundial con las que se implementan a nivel estatal, tomaremos los ejemplos de Estados Unidos y Argentina.

Luego, analizaremos las diferentes propuestas que se han ideado para dar respuesta a la problemática de la extinción y el riesgo de pérdida de la biodiversidad. Sobre el punto, estudiaremos la conveniencia del establecimiento de áreas protegidas, el funcionamiento de la Ley de Especies en Peligro de Extinción (ESA) de Estados Unidos y los mecanismos de incentivo y, por último, los centros de reproducción en cautividad y las biotecnologías reproductivas.

Ante este escenario, advertimos las dificultades burocráticas que deben superarse al momento de llevar a cabo algún proyecto que pretenda la recuperación y recomposición de la vida silvestre en riesgo. Para ilustrar esta situación tomaremos los casos del lobo mexicano en Estados Unidos y el huemul en Argentina.

\section{CLASIFICACIÓN LEGAL DE LAS ESPECIES: LAS CATEGORÍAS DE "VULNERABLE", "AMENAZADA" Y "EN PELIGRO DE EXTINCIÓN"}

La Unión Internacional para la Conservación de la Naturaleza (UICN) es una unión de miembros compuesta por Estados soberanos, agencias gubernamentales y organizaciones de la sociedad civil que tiene como objetivos asegurar la conservación de la naturaleza, especialmente de la diversidad biológica, como fundamento esencial para el futuro; asegurar que los recursos naturales sean utilizados en forma racional, equitativa y sustentable; y guiar al desarrollo de las comunidades humanas hacia modos de vida que sean tanto de buena calidad, como en armonía duradera con los otros componentes de la biosfera. ${ }^{4}$

Este organismo cuenta con un Programa Global de Especies ${ }^{5}$ y una Comisión de Supervivencia de Especies, cuya misión es conservar la diversidad biológica mediante el desarrollo y ejecución de programas destinados al estudio, salvaguarda, restauración y gestión acertada de las especies y sus hábitats, y, al mismo tiempo, proveen asesoramiento técnico y científico a proyectos de conservación en todo el mundo. ${ }^{6}$

\footnotetext{
Para mayor información acerca de la UICN, visitar https:/www.iucn.org/es/acerca-de-la-uicn

5 Para conocer más sobre las acciones y publicaciones llevadas a cabo por el Programa Global de Especies, visitar https:// www.iucn.org/es/tema/especies

6 Las actividades de la Comisión de Supervivencia de Especies pueden consultarse en https://www.iucn.org/theme/species/ about/species-survival-commission
} 
Actualmente, la UICN es la autoridad mundial en cuanto al estado de la naturaleza y los recursos naturales, así como las medidas necesarias para protegerlos. En este sentido, aporta mucha claridad al tema que nos ocupa, en tanto ha elaborado una lista roja de especies amenazadas, como inventario mundial que permite alertar al respecto del estado de la biodiversidad mundial y cuyas aplicaciones a nivel nacional permiten a los tomadores de decisiones considerar las mejores opciones para la conservación de las especies. ${ }^{7}$

Para elaborarla, la UICN utiliza categorías y criterios preestablecidos que tienen la intención de ser un sistema de fácil comprensión para clasificar especies en alto riesgo de extinción global, partiendo de la idea de que la extinción es un proceso estocástico. Así, adjudicar a un taxón una categoría de alto riesgo de extinción implica una expectativa más alta de extinción, y dentro del margen de tiempo considerado, es de esperar que se extinga un mayor número de taxones incluidos en una categoría de mayor amenaza, que aquellos que se encuentran en una de menor amenaza (en ausencia de actividades efectivas de conservación).

Sobre la base de estos parámetros de análisis, la categoría de especies "amenazadas" comprende tres niveles de peligro de extinción, tomando en consideración factores como el tamaño y reducción de la población, distribución geográfica (extensión de la presencia o área de ocupación) y probabilidad de extinción en estado silvestre:

- Especies "en peligro crítico": el tamaño de la población se estima en menos de 250 individuos maduros y/o el análisis cuantitativo muestra que la probabilidad de extinción en estado silvestre es de por lo menos el $50 \%$ dentro de 10 años o tres generaciones, es decir, un riesgo extremadamente alto de extinción.

- Especies "en peligro": el tamańo de la población se estima en menos de 2500 individuos maduros y/o el análisis cuantitativo muestra que la probabilidad de extinción en estado silvestre es de por lo menos $20 \%$ dentro de 20 años o cinco generaciones, es decir, un riesgo muy alto de extinción.

- Especies "vulnerables": el tamaño de la población se estima en menos de 10000 individuos maduros, una población muy pequeña o restringida y/o el análisis cuantitativo muestra que la probabilidad de extinción en estado silvestre es de por lo menos $10 \%$ dentro de 100 años, es decir, un riesgo alto de extinción. ${ }^{8}$

7 La página web de la UICN proporciona toda la información acerca de la lista Roja. Puede accederse visitando https:// www.iucnredlist.org/es/

8 Además de la categoría de especies "amenazadas", la UICN clasifica las especies en "extinto", "casi amenazado”, "preocupación menor" o "datos insuficientes". Para mayor información acerca de las categorías y criterios de listado, véase UICN, "Categorías y Criterios de la Lista Roja de la UICN: Versión 3.1", Comisión de Supervivencia de Especies de la UICN, UICN, Gland, Suiza y Cambridge, Reino Unido, 2001, https://www.iucn.org/es/content/categor\%C3\%ADas-y-criterios-de-la-lista-roja-de-la-uicn-versi\%C3\%B3n-31-segunda-edici\%C3\%B3n 
Simultáneamente, la Convención sobre el Comercio Internacional de Especies Amenazadas de Fauna y Flora Silvestres (CITES por sus siglas en inglés) regula el comercio de las especies amenazadas, con el objetivo de asegurar su supervivencia. Consta de tres apéndices en los cuales estas especies son clasificadas. ${ }^{9}$

- Apéndice I: se incluyen todas las especies en peligro de extinción. El comercio en especímenes de esas especies se autoriza solamente bajo circunstancias excepcionales.

- Apéndice II: se incluyen especies que no se encuentran necesariamente en peligro de extinción, pero cuyo comercio debe controlarse a fin de evitar una utilización incompatible con su supervivencia.

- Apéndice III: se incluyen especies que están protegidas al menos en un país, el cual ha solicitado la asistencia de otras Partes en la CITES para controlar su comercio. ${ }^{10}$

\subsection{Criterios de clasificación de Estados Unidos}

Ahora bien, además de las sistematizaciones facilitadas por los organismos internacionales, cada Estado regula la protección y conservación de sus especies autóctonas de manera independiente.

Estados Unidos no incluye el reconocimiento del derecho al ambiente sano en una norma expresa de su Constitución, sino que se trata de un derecho implícito. Sin embargo, cuenta con un "Sistema de Directrices" emanado del Servicio Nacional de Parques que cumplen un rol preponderante en la política pública y en la gestión en materia de conservación de las unidades concretas. Conjuntamente, existe legislación de carácter ambiental, aplicable en toda la administración. En relación al tema que nos ocupa, sirvan como ejemplo la Ley Nacional de Protección Ambiental de 1969 — National Environmental Protection Act—, el Fondo para la conservación de espacios terrestres y acuáticos y la Ley de áreas silvestres.

En especial, la Ley de Especies en Peligro de Extinción —Endangered Species Act (ESA)— de 1973 tiene como objetivo restaurar las especies en peligro de extinción mediante la conservación de sus ecosistemas y la elaboración de planes de recuperación. Para ello, exige a las agencias federales que el desarrollo de sus actividades no genere impactos desfavorables sobre las especies amenazadas o en peligro, así como tampoco modifique negativamente sus hábitats críticos. ${ }^{11}$

9 Mayor información acerca de la CITES en https://cites.org/esp/disc/how.php

10 Para conocer más en relación a la clasificación en "Apéndices" de la CITES, visitar https://cites.org/esp/app/index.php

11 "Hábitat crítico" se define como el espacio "esencial para la conservación de la especie", ya sea que la especie amenazada o en peligro de extinción habite en el área en el momento o no. 
A fin de determinar qué especies merecen protección, el Secretario del Servicio de Pesca y Fauna Silvestre - Fish and Wildlife Service (FWS) - , utilizando un criterio poco analítico o descriptivo, prepara una lista de especies amenazadas y en peligro de extinción:

- Una "especie amenazada" es cualquier especie que es probable que se convierta en una especie en peligro de extinción en el futuro previsible en todo o una significativa parte de su rango.

- Una "especie en peligro de extinción" es cualquier especie que se encuentre en peligro de extinguirse en todo o una parte significativa de su área de distribución distinta a una especie de la Clase Insecta que el Secretario determine que constituye una plaga cuya protección, según las disposiciones de esta Ley, presentaría un riesgo abrumador e importante para el hombre.

A partir de esta clasificación, sin importar si las especies se encuentran en propiedad privada, se establece la prohibición de "hostigar, dañar, perseguir, cazar, disparar, herir, matar, atrapar, capturar o recolectar, o intentar participar en cualquiera de tales conductas". La ley no define lo que se entiende por "daño", pero el FWS ha dicho que incluye "modificación o degradación significativa del hábitat que mata o lesiona la vida silvestre al afectar significativamente los patrones de comportamiento esenciales, como la reproducción, alimentación o refugio". ${ }^{12}$

\subsection{Criterios de clasificación de Argentina}

A fin de elaborar las clasificaciones a nivel interno, los Estados también deberán adecuarse a los compromisos adoptados a través de tratados y declaraciones. Al solo efecto de citar algunos de los más importantes, vale traer a colación los instrumentos surgidos a partir de la "Cumbre de Río" de 1992: la Agenda 21, la Declaración de Río sobre el Medio Ambiente y el Desarrollo, la Declaración de Principios relativos a los Bosques, la Convención Marco de las Naciones Unidas sobre el Cambio Climático y el Convenio sobre la Diversidad Biológica (CDB). En particular, el objetivo de este último es la conservación de la biodiversidad, el uso sostenible de sus componentes y la participación justa y equitativa de los beneficios resultantes de la utilización de los recursos genéticos. En relación con ello, en 2010, las Partes en el Convenio adoptaron el Plan Estratégico para la Diversidad Biológica 2011-2020, un marco de acción decenal para que todos los países e interesados salvaguarden la diversidad biológica y los beneficios que proporciona a las personas. Como parte del Plan Estratégico, se adoptaron 20 ambiciosas pero realistas metas, conocidas como las Metas de Aichi para la Diversidad Biológica.

12 La definición de los términos utilizados por la FWS puede conocerse en https://www.fws.gov/endangered/esa-library/ pdf/HCP_Handbook-Glossary.pdf 
Argentina ${ }^{13}$ es uno de los países con mayor adhesión a convenios y declaraciones sobre la protección del medio ambiente y sus postulados son tenidos en consideración al momento de elaborar la normativa interna.

El país cuenta con una profusa plataforma legal en materia ambiental, encabezada por el Art. 41 de la Constitución Nacional, que establece el derecho a un ambiente sano, equilibrado y apto para el desarrollo humano, y prevé la preservación del patrimonio natural y cultural y de la diversidad biológica, entre otras cosas. En relación al tema que nos atañe, también vale mencionar la Ley General de Ambiente y la Ley de Presupuestos Mínimos de Protección Ambiental de los Bosques Nativos. ${ }^{14}$

Además, la fauna silvestre que temporal o permanentemente habita el territorio de la República, así como su protección, conservación, propagación, repoblación y aprovechamiento racional ha sido declarada de interés público a través de la ley 22.421 .

Utilizando las mismas categorías que la UICN pero recurriendo a criterios de clasificación más generales — con menor base cuantitativa_- el Decreto 666/97 de Protección y conservación de la fauna silvestre propone la siguiente sistematización:

- Especies en peligro de extinción: aquellas especies que están en peligro inmediato de extinción y cuya supervivencia será improbable si los factores causantes de su regresión continuar actuando.

- Especies amenazadas: aquellas especies que por exceso de caza, por destrucción de su hábitat o por otros factores son susceptibles de pasar a la situación de especies en peligro de extinción.

- Especies vulnerables: aquellas especies que debido a su número poblacional, distribución geográfica u otros factores, aunque no estén actualmente en peligro, ni amenazadas, podrían correr el riesgo de entrar en dichas categorías. ${ }^{15}$

El Decreto también dispone que las especies que se hallaren amenazadas de extinción o en grave retroceso numérico, deberán ser protegidas adecuadamente para asegurar su conservación y propagación. La autoridad de aplicación promoverá y coordinará planes y programas tendientes a asegurar la protección de estas especies, como así también de su hábitat específico cuando ello sea necesario.

13 Vale la pena recordar que en relación con la jerarquía del derecho internacional, Estados Unidos de Norteamérica es el modelo clásico del dualismo en la relación con su derecho interno.

14 También existe la Ley 26.562 de Presupuestos Mínimos de Protección Ambiental para Control de Actividades de Quema en todo el Territorio Nacional y la Ley de Presupuestos Mínimos para la Preservación de los Glaciares y del Ambiente Periglacial.

15 El Decreto 666/97 también cuenta con las categorías de Especies no amenazadas y Especies insuficientemente conocidas. 
Por último, debemos referirnos a la ley 22.351 de Parques Nacionales, que ha instituido la categoría de "monumento natural" para designar áreas, cosas, especies vivas de animales o plantas, de interés estético, valor histórico o científico, a los cuales se les acuerda protección absoluta. Serán inviolables, no pudiendo realizarse en ellos o respecto a ellos actividad alguna (Art. 8). En la misma línea, el Convenio Internacional sobre la Diversidad Biológica ${ }^{16}$ determina que la ausencia de esos animales "significaría una desestabilización en la composición y estructura ambiental" del territorio.

\section{MECANISMOS IDEADOS PARA FOMENTAR LA CONSERVACIÓN Y RECUPERACIÓN DE LAS ESPECIES AMENAZADAS Y EN PELIGRO DE EXTINCIÓN}

A nivel mundial se han desarrollado diferentes mecanismos para hacer frente a la situación de vulnerabilidad y peligro en la que se encuentran las especies a las que nos referimos anteriormente.

A continuación, señalaremos las características de algunas de ellas, haciendo hincapié en las ventajas y desventajas de su utilización.

\subsection{Establecimiento de áreas protegidas}

La mejor estrategia de conservación de la biodiversidad es la preservación del medio natural.

El Convenio sobre la Diversidad Biológica plantea el concepto de "conservación in situ" como la conservación de los ecosistemas y los hábitats naturales y el mantenimiento y recuperación de poblaciones viables de especies en sus entornos naturales y, en el caso de las especies domesticadas y cultivadas, en los entornos en que hayan desarrollado sus propiedades específicas (Art. 2).

En su nuevo documento "Directrices para la aplicación de las categorías de gestión de áreas protegidas", la UICN define a las áreas protegidas como "un espacio geográfico claramente definido, reconocido, dedicado y gestionado, mediante medios legales u otros tipos de medios eficaces para conseguir la conservación a largo plazo de la naturaleza y de sus servicios ecosistémicos y sus valores culturales asociados". El término "área protegida”, entonces, engloba una extensa gama de zonas terrestres y marinas, es decir, una importante diversidad de enfoques de gestión, desde franjas fuertemente protegidas hasta fracciones donde el acento se focaliza en la conservación, pasando por modelos sumamente restrictivos en los que la preservación se completa con una extracción limitada y sostenible de los recursos.

16 Argentina es parte del Convenio sobre la Diversidad Biológica desde 1995. A través del siguiente enlace puede accederse a un perfil sobre las medidas adoptadas por el país en relación a los compromisos asumidos: https://www.cbd.int/countries/?country=ar. 
La creación de redes de áreas protegidas extensas, ecológicamente representativas, gestionadas eficazmente y financieramente seguras es una estrategia crítica, no solo para la conservación de la diversidad biológica, sino también para asegurar los bienes y servicios de los ecosistemas, permitir la mitigación y adaptación al cambio climático.

\subsection{El sistema de áreas protegidas en Estados Unidos}

Uno de los principales modelos para la creación de los sistemas de áreas protegidas sin duda lo constituye el Servicio Nacional de Parques de los Estados Unidos de América, dado que fue en este país donde se estableció el primer parque nacional. Se trata de un parámetro de referencia para el resto de las modalidades existentes en el derecho comparado al momento de determinar un régimen legal para la conservación de la naturaleza, ya sea por las capacidades técnicas de ese organismo rector, por los recursos económicos con los que cuenta o bien por la extensión del sistema nacional de referencia.

El sistema de parques nacionales de los Estados Unidos - National Park System-es un régimen federal de áreas protegidas que abarca el conjunto de unidades gestionadas por el $\mathrm{Na}$ tional Parks Service. Comprende los espacios clasificados como parques nacionales - National Parks - y la mayoría de los monumentos nacionales — National Monuments—, así como varias categorías de protección más, entre las que se destacan los memoriales, los parques militares, las áreas de recreo, las riberas y los senderos nacionales, entre otros. Actualmente manejan 418 unidades (generalmente referidas como "parques") y 150 áreas relacionadas. ${ }^{17}$

\subsection{El sistema de áreas protegidas en Argentina}

En la órbita del Medio Ambiente y Desarrollo Sustentable de la Nación se encuentra emplazada la Administración de Parques Nacionales — regulada a través de la Ley No 22.351 y el Decreto $\mathrm{N}^{\circ} 2148 / 90$ - Esta es la autoridad que controla los espacios encuadrados dentro de las categorías Parque Nacional, Monumento Natural, Reserva Nacional y Reserva Natural Estricta. ${ }^{18}$ Luego, cada provincia tiene establecidas por ley las categorías de gestión en que se clasifican las áreas protegidas de su jurisdicción.

En el país son cuatro las especies que entran en la categoría de "monumentos naturales": el huemul — sobre el cual nos explayaremos más adelante-, la taruca, el yaguareté y la ballena franca austral, que están en peligro de extinción, pero con escenarios diversos. ${ }^{19}$

17 Para mayor información respecto del National Park System, visitar https://www.nps.gov/aboutus/national-park-system. htm

18 Para mayor información respecto de las áreas protegidas argentinas, visitar https://www.parquesnacionales.gob.ar/ areas-protegidas/

19 Para mayor información respecto de los monumentos naturales argentinos, visitar https://www.parquesnacionales.gob. ar/areas-protegidas/monumentos-naturales/ 
Además, Argentina suscribió en 2011 el Decenio de las Naciones Unidas sobre la Biodiversidad, donde se exige que para 2020 las áreas protegidas de los países deben llegar al 17 $\%$ de su territorio terrestre y al $10 \%$ del ámbito marino. Hasta febrero de 2017, existían en el país 444 áreas protegidas con una superficie de 33956150 hectáreas que representaban el $12,16 \%$ del ámbito terrestre y $42503 \mathrm{~km}^{2}$ marinos protegidos, que representaban el 2,8 \% de la zona económica exclusiva. ${ }^{20}$

Sin embargo, en diciembre de 2018, la Cámara de Senadores sancionó, en el marco del Sistema Nacional de Áreas Marinas Protegidas — regulado a través de la Ley No 27.037-, la ley que crea las áreas marinas protegidas Yaganes y Banco Burdwood II. Su incorporación aporta más de 100 mil km² a la superficie marina protegida, constituyendo el primer paso para lograr la protección del $10 \%$ de los espacios marítimos argentinos, según lo previsto en el Plan Estratégico para la Diversidad Biológica 2011-2020. Con este logro, en 2018 resultan seis las nuevas áreas protegidas nacionales creadas en las provincias de Córdoba, Tucumán, Buenos Aires, Corrientes y el Mar Argentino Austral. ${ }^{21}$

\subsection{Restricciones a la propiedad privada y mecanismos de incentivo: la Ley de Especies en Peligro de Extinción de EE.UU.}

Tal como se adelantó en el apartado anterior, la Ley de Especies en Peligro toma como base la idea de mantener la propiedad en su estado natural, negando a los propietarios todo uso económico del espacio. La ESA les prohíbe degradar o destruir el hábitat y, por lo tanto, disuade el comportamiento dañino, pero no hace nada que obligue a realizar acciones útiles para la conservación. ${ }^{22}$

Empero, la regulación solo se aplica a los hábitats críticos actuales de la lista de las especies en peligro de extinción. No hace nada para evitar que los propietarios destruyan de forma preventiva los hábitats de las especies antes de la inclusión en la lista y no requiere que ellos hagan su tierra viable para constituir el hábitat de las especies después del listado, ${ }^{23}$ tornándose ineficaz en la promoción de la conservación en terrenos privados.

Frente a este escenario, los mecanismos de incentivo ofrecen una alternativa prometedora al mecanismo de mando y control de la ESA:

20 Esta información fue obtenida a través de las entrevistas plasmadas en la nota periodística "Expertos piden más áreas protegidas para frenar la pérdida de especies en peligro de extinción”. Disponible en: https://www.telam.com.ar/notas/201702/179312-expertos-piden-mas-areas-protegidas-para-frenar-la-perdida-de-especies-en-peligro-de-extincion. html

21 Junto con las áreas marinas protegidas Yaganes y Banco Burdwood II mencionadas, en 2018 se crearon los Parques Nacionales Traslasierra, Aconquija, Ciervo de los Pantanos e Iberá. Al respecto, visítese https://www.parquesnacionales.gob. ar/2018/12/2018-record-historico-en-creacion-de-parques-nacionales/

22 N. Paulich, "Increasing Private Conservation through Incentive Mechanisms", Stanford Journal of Animal Law \& Policy 3 (2010): 123, https://law.stanford.edu/wp-content/uploads/2018/05/paulich.pdf, (traducción propia)

23 Idem, 117 (traducción propia). 
- Planes de Conservación del Hábitat — Habitat Conservation Plans (HCP) —: se encuentran previstos dentro de la Ley (Sección 10) y están diseñados para "minimizar y mitigar el daño potencial causado a especies amenazadas y en peligro de extinción como resultado de un proyecto de desarrollo". Los planes, que se negocian entre el gobierno y los terratenientes, incluyen preparar un plan para minimizar y mitigar cualquier daño a una de las especies listadas y continuar monitoreando su población. ${ }^{24}$

- Acuerdos de Puerto Seguro —Safe Harbor Agreements (SHA) —: los propietarios voluntariamente entran en este acuerdo con el Servicio de Pesca y Fauna Silvestre para "restaurar, mejorar o crear hábitats" en beneficio de una especie enlistada. Acuerdan mejorar las condiciones de una especie durante un período específico de tiempo y puede devolver la propiedad a su línea de base al final del acuerdo. Los SHA tienen el efecto positivo de promover la conservación sin enajenar a los terratenientes de su propiedad. $^{25}$

- Acuerdos de Conservación de Candidatos - Candidate Conservation Agreements (CCA) —: los propietarios acuerdan voluntariamente emprender esfuerzos de conservación por especies candidatas o que puedan ser listadas en el futuro. La esperanza detrás de los CCA es que los esfuerzos proactivos de conservación harán innecesario el listado en el futuro. ${ }^{26}$

- Programas de compensación: Pueden establecerse a través del pago por adquisición simple de los derechos del terrateniente, subsidios o servidumbres de conservación. A cambio, el propietario puede recibir beneficios fiscales. Esto alentará a los propietarios a tomar en cuenta el valor económico y ecológico de sus tierras y puede realmente aumentar la cooperación en los esfuerzos de conservación y conducir a un uso más eficiente y efectivo de las tierras privadas. ${ }^{27}$

- Enfoques basados en el mercado: Los propietarios son recompensados por sus esfuerzos para ayudar a la conservación de las especies. Pueden ser a través de bancas de cotización (a partir de créditos otorgados en razón del hábitat y el número de especies encontradas) y la venta de derechos de desarrollo negociables con zonificación.

24 Idem, 132/133 (traducción propia). Para un estudio más profundizado de este tema, recomendamos la lectura de J. B. Ruhl, "How to Kill Endangered Species, Legally: The Nuts and Bolts of Endangered Species Act 'HCP' Permits for Real Estate Development", The Environmental Lawyer 5 (1998/1999), https://www.researchgate.net/publication/228137717_ How_to_Kill_Endangered_Species_Legally_The_Nuts_and_Bolts_of_Endangered_Species_Act_HCP'_Permits_for_ Real_Estate_Development

25 N. Paulich, "Increasing..., 135/136 (traducción propia).

26 Idem, 136 (traducción propia).

27 Idem, 138/139 (traducción propia). 


\subsection{Centros de reproducción en cautividad y biotecnologías reproductivas}

Existen situaciones en las que el deterioro de una especie se ha dado por un exceso de caza (por la actividad de furtivos), o por problemas sanitarios en la especie en cuestión o en la fuente de su alimentación. Por estos motivos, es importante reconocer que existen estrategias complementarias de conservación de biodiversidad. Entre ellas se incluyen la cría en cautividad, el desarrollo de bancos de recursos genéticos, y el uso de biotecnologías reproductivas (también llamadas de reproducción asistida). ${ }^{28}$

Al respecto, el Convenio sobre la Diversidad Biológica acuña el término de "conservación ex situ" para referirse a la conservación de componentes de la diversidad biológica fuera de sus hábitats naturales. En este escenario, "cada parte contratante, en la medida de lo posible y según proceda, y principalmente a fin de complementar las medidas in situ (...) establecerá y mantendrá instalaciones para la conservación ex situ y la investigación de plantas, animales y microorganismos, preferiblemente en el país de origen de recursos genéticos (...) y adoptará medidas destinadas a la recuperación y rehabilitación de las especies amenazadas y a la reintroducción de estas en sus hábitats naturales en condiciones apropiadas (...)”(Art. 9).

En primer lugar, a partir de los centros de recría se persigue alcanzar un tamaño de población suficientemente grande como para permitir, mediante proyectos de reintroducción o de reforzamiento, el restablecimiento de la especie en una o varias de las zonas en las que históricamente se distribuían sus poblaciones.

En estas circunstancias, la reproducción en cautividad de especies muy amenazadas se deberá enfrentar a limitaciones en el número de individuos disponibles, a problemas de reducida variabilidad genética y sus consecuencias negativas sobre la futura viabilidad y reproducción de estos individuos, y a los inciertos resultados de posibles reintroducciones.

Por otro lado, por medio de la biotecnología moderna, se modifica el material genético de un organismo con la finalidad de desarrollar o mejorar una o más características del organismo.

En términos de regulación internacional, adicionalmente al Convenio sobre Diversidad Biológica, el Protocolo de Cartagena sobre Seguridad de la Biotecnología busca garantizar la transferencia, manipulación y utilización seguras de los organismos vivos modificados (OVM) resultantes de la biotecnología moderna que puedan tener efectos adversos para la diversidad biológica, teniendo también en cuenta los riesgos para la salud humana. ${ }^{29}$

28 E. Roldán, J. Garde, "Biotecnología de la reproducción y conservación de especies en peligro de extinción”, en Los retos medioambientales del siglo XXI. La problemática de la conservación de la biodiversidad en España, ed. por Montserrat Gomendio Kindelán (España: Fundación BBVA), 307-338, http://www.gebir.csic.es/descargas/Roldan_Garde_ReproTech_FBBVA.pdf

29 El Protocolo establece procedimientos para regular la importación y exportación de OVM de un país a otro. Hay dos 
Un ejemplo de la utilización de la biotecnología es la creación de células madre a partir de dos especies en peligro de extinción, que podrían servir para asegurar su supervivencia, en tanto la clonación no ha funcionado bien para estas especies. ${ }^{30}$

Sea cual sea el método utilizado, la reintroducción de las especies implica que el medio ambiente siga siendo capaz de acoger esta nueva población y también que los habitantes de las regiones afectadas acepten su presencia. Una condición de que no siempre es fácil de obtener, especialmente en el caso de especies depredadoras (lobo, lince) o que puedan causar daños a los cultivos (elefantes en África). ${ }^{31}$

\section{LA BUROCRACIA COMO OBSTÁCULO HACIA LA RECUPERACIÓN Y CONSERVACIÓN DE ESPECIES AMENAZADAS Y EN PELIGRO DE EXTINCIÓN}

Los mecanismos de recuperación y conservación mencionados previamente se encuentran hundidos en complejos procesos que implican numerosas leyes, convenios y personal, así como un presupuesto elevado y mucho tiempo de inversión. Además, en varios países, por causa de las crisis se han aplicado recortes que han afectado los Ministerios encargados de los planes de gestión referidos a la biodiversidad. Esto conlleva medidas de ahorro importantes y a veces catastróficas para muchas especies.

En este sentido, si bien las áreas protegidas son grandes herramientas para la ideal conservación in-situ de la especie, estas resultan frecuentemente vulneradas o presentan serios problemas de administración. Valga de ejemplo lo que sucedió en 2016 cuando un huemul macho había aparecido, sano y sin heridas, en la zona de El Manso. Fue atado por un poblador y un guardaparque quiso soltarlo, pero el intendente del Parque Nahuel Huapi ordenó esperar a que lleguen los "expertos y especialistas" a analizar al animal.

Éstos arribaron 14 horas después de encontrado el espécimen, pero sin el equipamiento necesario, ni sedantes, ni radio collares, y ni siquiera llevaron el kit básico de emergencias, según sus propias palabras. Además, los expertos expusieron que carecían de un protocolo y de presupuesto. Tras varias horas de idas y vueltas, el animal murió al día siguiente.

conjuntos principales de procedimientos, uno para OVM que esté previsto introducir directamente en el medio ambiente, conocido como acuerdo fundamentado previo (AFP), y otro para aquellos que estén destinados para uso directo como alimento humano o animal o para procesamiento (OVM-AHAP). El Protocolo de Cartagena fue luego reforzado por el Protocolo de Nagoya-Kuala Lumpur Suplementario sobre Responsabilidad y Compensación.

30 "Nueva esperanza para las especies en peligro de extinción", nota periodística del 5 de septiembre de 2011, https://www. bbc.com/mundo/noticias/2011/09/110904_ciencia_celulas_madre_salvacion_especies_peligro_extincion_jrg

31 J. Espunyes Nozières, Reintroducción... 
Más de dos años después de la muerte del huemul, la APN no cumplió con la resolución del caso, pese a la promesa de los tres meses y no informó oficialmente absolutamente nada del tema. ${ }^{32}$

Por otro lado, la estructura actual de mando y control de la ESA impone costos sustanciales en los propietarios y, consecuentemente, crea desincentivos para que estos conserven las especies que se encuentran en su terreno y los alienta a destruir los hábitats que la ley está destinada a proteger.

El proceso de listado que dispone la ESA es el evento más crítico para la protección de las especies. El secretario de la FWS tiene amplia discreción para llevarlo a cabo y debe basar la decisión únicamente en factores científicos que ignoran el impacto económico potencial en el sector privado. ${ }^{33}$

En la práctica, la ESA implica una complicada intersección entre análisis científico y legal. Los estándares legales de la ley requieren determinaciones que los científicos suelen ser reacios a hacer, y la información y análisis que produce la ciencia a menudo conducen a resultados no concluyentes a la luz de los patrones legales. La imperfección del sistema lo deja susceptible a ser manipulado por los procesos políticos y grupos que tienen intereses en contra de la ESA. ${ }^{34}$

En este contexto, los mecanismos de incentivo se presentan como una buena alternativa a un modelo enteramente regulatorio, pero no se encuentran exentos de trabas burocráticas que afectan sus posibilidades de éxito. Esto es así, ya que los programas de compensación terminan siendo caros, en tanto requieren monitoreo y aplicación para garantizar que los propietarios cumplan lo pactado. Asimismo, establecer un mercado para la comercialización de derechos de desarrollo conlleva procedimientos complejos y administrativamente difíciles.

En relación a los procesos de cría en cautividad, existe una relación entre la aceptación de la conservación ex situ y el número de individuos de una especie. La aceptación de un centro de conservación es muy alta, y no preocupa cuando la especie es abundante; se empieza con centros cuando una especie todavía no ha llegado a estar en peligro. No conviene en absoluto esperar para comenzar la instalación de centros hasta que solo queden muy pocos individuos. Esta parálisis o miedo a la intervención directa resulta significativa en los administradores de fauna silvestre local. ${ }^{35}$

32 "A un año de la muerte del huemul, parques no determinó responsables ni causas del deceso", nota periodística del 20 de marzo de 2017, https://www.barilochense.com/notas/a-un-ano-de-la-muerte-del-huemul-parques-no-determino-responsables-ni-causas-del-deceso

33 N. Paulich, "Increasing..., 113 (traducción propia).

34 Idem, 112 (traducción propia).

35 E. Escobar Ruiz, J. Smith, W. Flueck, "El Huemul. Shoonem. Madera que se mueve/re”, Fundación SHOONEM, 2018, https://deerlab.org/tmp/Escobar2018libroHuemul.pdf 
En la misma línea, las críticas a la utilización de la biotecnología de la reproducción y la dificultad de acceder y manejar las especies en peligro de extinción, con el consecuente desconocimiento de aspectos básicos de su biología, imposibilitan el aprovechamiento al máximo de esta novedosa alternativa.

\section{EL CASO DEL LOBO MEXICANO EN ESTADOS UNIDOS ${ }^{36}$}

El caso del lobo mexicano resulta ejemplificativo de lo que se ha denominado una especie "burocráticamente en peligro". Esto sucede cuando los intereses económicos o la oposición ideológica se conjugan con la colaboración o complacencia de la agencia pública en el bloqueo del progreso genuino hacia la conservación de las especies, en los términos de la ESA.

El lobo mexicano fue incluido en la lista de especies en peligro de extinción de la ESA en 1976 y a partir de ese momento se creó un área de recuperación — the Blue Range Wolf Recovery Area - a fin de establecer una población mínima. Sin embargo, surgieron importantes restricciones políticas que han dificultado la capacidad del programa para liberar lobos donde más se necesitan, es decir, en hábitats de alta calidad que carecen de lobos, o para reemplazar las parejas perdidas y proveer a la mejora genética.

Como si ello fuera poco, más adelante se incluyeron restricciones adicionales a la recuperación de los lobos, por iniciativa de un comité interinstitucional de supervisión de gestión adaptativa - Adaptive Management Oversight Committee - que propuso eliminar permanentemente a los lobos que depredan el ganado o crean impactos inaceptables en las poblaciones nativas si la población del lobo llegaba a 125 individuos.

Además, se ha planificado desarrollar los planes de recuperación en zonas distintas al rango geográfico central de la especie, siendo que dicho enfoque amenaza el carácter distintivo del lobo mexicano en violación del requisito de la ESA de conservar las subespecies, ya que no se estaría considerando que la biología evolutiva se vería afectada por el aislamiento por distancia, clima y hábitat a escala continental.

Sumado a esto, en atención a que el lobo mexicano habita en la frontera entre Estados Unidos y México, las estrategias deben ser coordinadas. Al respecto, las prioridades de México para esta especie incluyen la reintroducción, conservación del hábitat, consolidación de un sistema de áreas de vida silvestre, acuerdos de conservación con los ganaderos, agricultores y mineros, y una mayor colaboración con los Estados Unidos.

36 A. Povilitis, D. Parsons, M. Robinson, D. Becker, "The Bureaucratically Imperiled Mexican Wolf", Conservation Biology, Society for Conservation Biology 20, n. ${ }^{\circ} 4$ (2006), https://www.researchgate.net/publication/6866374_The_Bureaucratically_Imperiled_Mexican_Wolf. https://doi.org/10.1111/j.1523-1739.2006.00489.x PMid:16922210 
Todos estos puntos dejan al descubierto que la gestión manipulativa ineficaz y el abandono de proyectos de conservación del ecosistema en deferencia a los intereses económicos o ideológicos ponen en peligro los programas de recuperación de la vida silvestre.

\section{EL CASO DEL HUEMUL EN ARGENTINA}

A modo introductorio, a fin de comprender el trayecto administrativo al que debe enfrentarse un proyecto de protección de especies en peligro de extinción, nos referiremos a la estructura orgánica argentina.

El organismo encargado de velar por estas especies es el Ministerio de Medio Ambiente y Desarrollo Sustentable, del cual depende el Programa de Conservación de Especies Amenazadas, que está organizado en distintos proyectos que se basan en la realización de acciones para la preservación de especies y sus hábitats, tales como la concientización del público general y grupos interesados sobre la situación de las especies, la disminución de la presión de caza, la generación de estrategias de conservación de sus hábitats, actividades de rescate y reintroducción al medio silvestre, propuestas de estudio, entre otras. Estas acciones se plantean conjuntamente con las provincias en las cuales estas especies tienen distribución, fundamentalmente tendiendo a la toma de decisiones consensuadas.

En 2016 el programa fue reemplazado por el Plan Extinción Cero que, aunque no cuenta con una resolución de creación que lo establezca, ha ocupado su lugar teniendo como objetivo fortalecer las acciones y políticas para la conservación de especies en estado crítico. ${ }^{37}$ Además, se ha adoptado la Estrategia Nacional sobre la Biodiversidad y Plan de Acción 20162020 (ENBPA) que consiste en la formulación e instrumentación de políticas, iniciativas, normativas y procedimientos que, en forma coordinada, promuevan un mayor conocimiento de los bienes y servicios ambientales, la conservación y protección de la biodiversidad y su utilización en un marco de desarrollo sustentable. ${ }^{38}$

Establecido el marco estructural, es importante remarcar que en la Argentina hay 564 especies de fauna silvestre en peligro de extinción, amenazadas y vulnerables. En particular, el caso del huemul es trascendental, ya que se lo considera una especie "paraguas" cuya conservación ofrecería protección a una amplia variedad de otras especies. ${ }^{39}$

37 Para conocer más acerca del Programa "Extinción Cero", visitar https://www.argentina.gob.ar/ambiente/biodiversidad/ extincioncero

38 Para conocer más acerca del ENBPA, visitar https://www.argentina.gob.ar/ambiente/biodiversidad/estrategianacional

39 A. Povilitis, "El estado actual del huemul (Hippocamelus bisulcus) en Chile central", Gayana (Concepc.) [online] 66, nº 1 (2002): 59-68, https://scielo.conicyt.cl/scielo.php?script=sci_arttext\&pid=S0717-65382002000100008\#1 https://doi.org/10.4067/S0717-65382002000100008 
Tanto Argentina como Chile comparten el huemul, su hogar y el desafío de su protección. Nadie sabe con certeza cuántas pequeñas familias de estos ciervos en la actualidad se refugian en la cordillera patagónica argentino-chilena, aunque se estima que unos 600 ejemplares viven en nuestro territorio nacional. ${ }^{40}$

El huemul ha sido declarado en peligro de extinción por la Resolución SAyDS 1030/04 y monumento natural por ley 24.702. ${ }^{41}$ Las provincias de Santa Cruz (Ley 2103/89), Chubut (Ley 4.793/01) y Río Negro (Ley 2.646/93) también le otorgaron esta última figura de protección legal. En Chile también está considerado "en peligro de extinción”. Entre las amenazas se mencionan: competencia alimenticia y riesgos de transmisión de nuevas enfermedades a partir de la introducción y liberación de ciervos europeos, destrucción y fragmentación del hábitat por la acción directa del ramoneo y pastoreo del ganado afectando la composición y estructura de la vegetación y, en los lugares de alta carga ganadera, disminuyendo drásticamente la regeneración del bosque nativo; y por otro lado, por la acción humana al crear o aumentar áreas de pasturas para el ganado eliminando zonas arbustivas y boscosas (principalmente con la ayuda del fuego). La caza también es un factor de amenaza importante. ${ }^{42}$

Mundialmente se encuentra clasificado dentro del apéndice I de la CITES —el cual prohíbe toda acción de comercio internacional de sus productos- y en el Apéndice I de la Convención sobre la Conservación de las Especies Migratorias de Animales Silvestres (CMS), por tratarse de una especie compartida entre dos países y que se desplaza habitualmente entre sus límites.

Del mismo modo, ha sido calificada como "en peligro de extinción” por la UICN a razón de la baja cantidad de individuos maduros (menos de 2500), la tendencia a su disminución, la mengua de la extensión ocupada, la fragmentación de su población y la disminución continuada del área de ocupación, extensión, y calidad de hábitat y del número de subpoblaciones. ${ }^{43}$

La Administración de Parques Nacionales, las áreas protegidas provinciales y varias organizaciones no gubernamentales, sumadas a las instituciones transcordilleranas, trabajan para que el huemul —el fantasma de los Andes - no se vaya de nuestras montańas y de nuestra cultura. En este marco, desde el año 1992 se lleva a cabo el programa "Conservación del Huemul” de la Administración de Parques Nacionales, y se realizan las reuniones binacionales

40 La información que brinda la Administración de Parques Nacionales sobre el monumento natural huemul está disponible en https://www.parquesnacionales.gob.ar/areas-protegidas/monumentos-naturales/mn-huemul/

41 También ha sido declarada "en peligro de extinción" por el Consejo Asesor Regional Patagónico de Fauna Silvestre y ha sido confirmada por el Libro Rojo de Mamíferos Amenazados de Argentina y el Libro Rojo de los Vertebrados Terrestres de Chile.

42 Auditoría General de la Nación, "Informe de auditoría. Examen de la gestión del Programa de Conservación de Especies Amenazadas. Período auditado: del 01-01-2011 al 31-03-2016”, https://www.agn.gov.ar/files/informes/2018_062info.pdf

43 La información sobre el huemul como parte de la lista roja de la UICN puede conocerse en https://www.iucnredlist.org/ es/species/10054/22158895 
entre Chile y Argentina. ${ }^{44}$ Este programa surge como un programa a largo plazo con la meta de contar con un sistema organizado de relevamientos de áreas, ubicación y monitoreo de subpoblaciones o grupos de huemules dentro de los Parques Nacionales, e identificación y corrección de los problemas de conservación existentes. Para ello, contempla tareas de gestión, entrenamiento, educación, difusión, etc.

Sin embargo, la participación de las provincias patagónicas en las reuniones del programa muchas veces es incompleta, es decir los estados no envían representantes, y otras veces los representantes no conocen la temática aunque por estar encargados de organismos y reparticiones oficiales son responsables, y es común la no disponibilidad de presupuestos para estos menesteres. Al ser una temática que afecta a los dos estados nacionales, Argentina y Chile, en las reuniones de integración binacional y de frontera, la temática huemul tiene demoras burocráticas que no se condicen con la urgencia que impone la extinción. A las fuentes de información institucionales a las que se recurre son las que se mencionan y los organismos a cargo de la fauna en las provincias. ${ }^{45}$

Otra alternativa a la que se ha recurrido para propender a la conservación y recuperación de la especie es el establecimiento de áreas protegidas:

- El Parque Shoonem fue creado por la Municipalidad de Alto Río Senguer (Chubut) en el año 2013 y tiene como objetivos fundacionales: preservar el hábitat del huemul, facilitar un corredor biológico binacional, promover la actividad científica y educativa, mantener las condiciones naturales de los cuerpos lacustres, ríos y arroyos y prevenir su contaminación, promover el desarrollo turístico sustentable, prevenir el acceso de especies invasoras en los ecosistemas nativos, manejar el ambiente lacustre basados en criterios científicos y fomentar el desarrollo de la comunidad de Alto Río Senguer.

- El Plan de acción para la conservación del Huemul en el Parque Nacional Perito Moreno, aprobado en 2014, prevé la realización de patrullas periódicas en distintos sectores del área protegida, previamente seleccionados. Además de los objetivos específicos establecidos en el plan, la incorporación de personal de otras instituciones busca estrechar vínculos con las mismas, mostrar el trabajo que lleva adelante el personal del área protegida y difundir los valores naturales y culturales mediante la realización de recorridas en el terreno. ${ }^{46}$

44 Conforme información extraída de la página web de la Administración de Parques Nacionales. Anteriormente, en 1971, se había impulsado el Operativo Nacional Huemul para preservar de su total extinción al huemul, cuyo objetivo era capturar huemules y destinarlos a una estación zoológica donde el personal especializado se encargaría de lograr la reproducción, con vistas a incorporarlos a zonas intangibles o en reservas de los parques nacionales. Por motivos desconocidos el programa no se concretó.

45 E. Escobar Ruiz, J. Smith, W. Flueck, "El Huemul...

46 Para conocer más respecto del "Plan de acción para la conservación del Huemul”, visítese https://www.parquesnacionales. gob.ar/2016/03/plan-de-accion-para-la-conservacion-del-huemul/ 
- En 2015 fue creado el Parque Nacional Patagonia, que aumenta seis veces la continuidad de hábitat protegido para la importante población de huemules. La población del Valle Chacabuco/Tamango está estimada en 150 individuos, un porcentaje significativo de la población total de la especie. Al retirar el ganado, revivir los ecosistemas y al abrir hábitat para darle conectividad, el Parque le brinda al huemul un hábitat de gran calidad para recuperarse.

La creación de estos espacios protegidos ha generado buenos resultados, pero no ha alcanzado para recuperar la especie, en tanto los ejemplares que habitan estos espacios no se reproducen lo suficientemente rápido para recomponer la cantidad de ejemplares maduros necesarios para que los huemules dejen de estar en peligro.

Por otra parte, una opción con gran viabilidad, pero mucha resistencia política y administrativa es la reproducción en cautividad.

Un centro de recría puede concentrarse en dos objetivos básicos: conservación biológica y educación. Paralelamente puede servir para reforzar y abastecer las poblaciones amenazadas, o repoblar áreas que perdieron la especie, para rehabilitar huemules enfermos o lesionados, para mejorar la variabilidad genética, y para educación ambiental. ${ }^{47}$

La UICN desde 2008 se ha expresado respecto de los centros con huemules semicautivos para estudios y reintroducciones como una valiosa herramienta de recuperación, dado que permite estudios mediante reintroducciones basado en manejo adaptativo.

Además, ha manifestado explícitamente que estos centros ex situ con programas de propagación cautiva deben ser utilizados para fomentar la investigación y responder a preguntas relevantes concernientes a la conservación in situ. Para evaluar la conveniencia de un centro, además del tamaño total de la población, también hay que ponderar factores relevantes para la conservación como el gran vacío de información pertinente sobre la especie, la fragmentación, las tendencias poblacionales, los cambios de hábitat y de las fluctuaciones estocásticas del ambiente. ${ }^{48}$

En 1936 la Dirección de Parques Nacionales de Argentina inició un programa pionero con un centro de recría en Bariloche para evitar la extinción del huemul, con el objetivo final de poder reintroducirlo en áreas históricamente ocupadas. Este centro parecía tener buenas expectativas de resultados, pero fracasó cuando los huemules se escaparon accidentalmente y luego debió cerrarse por problemas económicos.

47 E. Escobar Ruiz, J. Smith, W. Flueck, "El Huemul...

48 Idem. 
Este plan pretende reflotarse a partir de la Estación de Recría para el Parque Shoonem que se ha autorizado en octubre de 2018. Su objetivo principal es la preservación y conservación de cérvidos autóctonos, el fomento de la recuperación de la especie, la promoción de actividades científicas y de manejo, programas de educación ambiental, divulgación de conocimientos científicos y formación de recursos humanos. A la fecha no se cuenta con información respecto a su funcionamiento.

Lo mismo sucede en el caso de la Provincia de Chubut, donde el Art. 12 de la Ley XI-10 contempla el rubro de Estación de Recría, pero aún no se ha puesto en práctica para el caso del huemul.

Los planes para establecer un centro de recría en Chile también han debido enfrentar inconvenientes. En 2005 se fundó el centro Huilo Huilo, al cual originalmente se pretendían trasladar 6 huemules como experiencia piloto, pero dada la controversia generada en la opinión pública solo fue posible trasladar una primera pareja. Actualmente está fracasando el pedido de traslocar animales para ampliar el bagaje genético del Centro, y se repite la resistencia institucional y pública.

Este cuadro de situación ha llevado a que en el caso de huemules en Argentina se produzca lo siguiente:

- Han llegado a un tamaño crítico de población hace décadas, justificando la implementación de programas ex situ.

- No hay en el territorio ninguna subpoblación de tamaño considerado viable según el estándar internacional.

- Los recientes y actuales esfuerzos de conservación fueron insuficientes para lograr algún nivel de garantía para la supervivencia de huemul a largo plazo.

- La conservación ex situ por lo tanto se ha vuelto esencial como herramienta adicional en comparación con las actividades in situ efectuadas hasta la fecha.

- Los riesgos asociados al cautiverio son mínimos basados en las experiencias históricas $\mathrm{y}$ actuales enunciadas.

- Varios casos previos y el actual centro de conservación Huilo Huilo certifican la factibilidad de tener huemules bajo condiciones controladas. ${ }^{49}$

49 E. M. Escobar Ruiz, Z. Gizejewski, S. Hoby, W. T. Flueck, J. M. Smith-Flueck, "El valor de los centros de conservación para la supervivencia del huemul”, en El Huemul de Aysén y otros rincones, ed. por A. Irarte, D. Donoso, B. Segura, M. Tirado (Chile: Ediciones Secretaría Regional Ministerial de Agricultura de la Región de Aysén y Flora y Fauna), 198215, https://www.researchgate.net/publication/319177637_EL_VALOR_DE_LOS_CENTROS_DE_CONSERVACION_PARA_LA_SUPERVIVENCIA_DEL_HUEMUL 


\section{CONCLUSIONES}

La conservación de la naturaleza debe ser una acción consciente y enfocada a la subsistencia de la especie humana y de su calidad de vida, en tanto la pérdida de biodiversidad no solo afecta a la productividad de los ecosistemas, sino que está generando alteraciones sociales y culturales importantes, relacionadas con el aprovechamiento y disfrute que el ser humano realiza del medio natural..$^{50}$

En las últimas décadas venimos observado con gran preocupación la aceleración del ritmo de desaparición de miles de especies así como la incesante alteración, degradación y pérdida de los ecosistemas en los que viven, que también forman parte de la biodiversidad biológica. ${ }^{51}$

La información de la Lista Roja indica que la fuente de nuestros alimentos, medicinas y agua potable, además de los medios de subsistencia de millones de personas, podrían estar en riesgo con la rápida disminución de las especies animales y vegetales del mundo.

El "orden público ambiental" se ha constituido —en los últimos años— en el núcleo duro del derecho ambiental a la luz de la doctrina judicial, que lo interpreta como un orden público de coordinación, en tanto las leyes ambientales armonizan las acciones individuales con ciertos valores esenciales que el ordenamiento jurídico decide proteger, Este nuevo orden público apunta al conjunto de normas imperativas que controla la licitud del ejercicio de los derechos individuales para hacer posible la vida social. ${ }^{52}$

Es poco común que se discuta sobre los modelos de justicia que fundamentan las normas y las estrategias políticas que pretenden proteger el ambiente. En estas coordenadas, el derecho ambiental se transforma en un conjunto de normas analizadas sin tener en cuenta el contexto en el que se aplican, ni su eficacia. Las exigencias actuales hacen que lo urgente tenga prioridad frente a lo que en apariencia se puede relegar, como es la fundamentación teórica de las acciones y de la normativa vigente..$^{53}$

Las medidas de protección y conservación descriptas en los parágrafos anteriores dejan al descubierto sus falencias. Si bien es cierto que no existe un único mecanismo de incentivo que sea ideal para todos, lo que ha quedado demostrado es que las trabas administrativas y burocráticas se convierten en un gran obstáculo al momento de poner en marcha las diferentes propuestas de recuperación y conservación.

50 OESA - Fundación Biodiversidad, Caracterización de la cría en cautividad y repoblación de especies de interés a través de la acuicultura (Madrid: Fundación Biodiversidad, 2018), https:/www.mapa.gob.es/app/jacumar/recursos_informacion/ Documentos/Publicaciones/338_Caracterizacion_cria_cautividad_repoblacion_especies_interes.pdf

51 J. Espunyes Nozières, Reintroducción...

52 A. Petrella, "La justicia ambiental a la luz del constitucionalismo social", LL, Buenos Aires, 2011-B-19.

53 D. Bonilla Maldonado, "Justicia ambiental, normas jurídicas y acción política", en Derecho ambiental y justicia social, comp. por G. Hardín, C. Stone, C. Rose, estudio preliminar de Colin Crawford (Bogotá: Siglo del Hombre Editores, 2009), 11-12. 
En este sentido, observamos que las previsiones de la ESA no brindan protección a especies ni imponen limitaciones a propietarios hasta que una especie supere el proceso de listado y consiga la categoría de "amenazada” o "en peligro de extinción”. Correlativamente, la destrucción preventiva del hábitat por efecto de la regulación impuesta por la ESA puede causar dańos a largo plazo en el hábitat y en las especies incluidas en la lista.

Los acuerdos de conservación y los mercados son una buena herramienta y tienen una influencia positiva en el medio ambiente, dado que reemplazan la toma de decisiones burocráticas con incentivos económicos para coordinar decisiones más eficientes por parte de actores privados. ${ }^{54}$ Empero, reposan en gran medida en la voluntad y predisposición de los propietarios, y no están exentos de controles y medidas regulatorias complejas.

Las áreas protegidas parecen ser siempre la mejor opción, en tanto salvaguarda los ecosistemas naturales, funciona como refugio para las especies y mantiene los procesos ecológicos que no son aptos para sobrevivir en entornos terrestres o marítimos con alto grado de intervención humana. No obstante, en ocasiones no resulta suficiente, como sucede con el caso de los huemules en la Patagonia. De hecho, en el año 2001 se advirtió que los parques nacionales en Argentina tal vez no contengan ninguna población viable. ${ }^{55}$

El Programa "Conservación del Huemul" creado para hacer frente al crítico cuadro de situación de esta especie fue auditado por la Auditoría General de la Nación en el período enero de 2011 a marzo de 2016, poniendo de manifiesto las graves falencias burocráticas que tenía.

Durante el trabajo de auditoría, el programa no remitió planificaciones estratégicas ni operativas anuales ni informes de gestión, así como tampoco documentación sobre la etapa de planificación del Plan de Extinción Cero. Aun así, la AGN encontró que:

- El programa no cuenta con lineamientos específicos ni metas concretas o cronogramas de cumplimiento, ni con criterios que justifiquen la selección de las especies trabajadas.

- De las 12 especies que el programa había seleccionado, solo 5 poseen plan o programa nacional/internacional de conservación.

- El Plan de Extinción Cero ha reducido el número de especies objeto de acciones de conservación de 12 a 7, vulnerando el principio de progresividad.

- Los recursos humanos son insuficientes.

54 N. Paulich, "Increasing..., 147 (traducción propia).

55 E. M. Escobar Ruiz, Z. Gizejewski, S., Hoby, W. T. Flueck, J. M. Smith-Flueck, "El valor de los centros de conservación para la supervivencia del huemul", en El Huemul de... 
La auditoría destacó que la falta de financiamiento es la principal causa para que las acciones de conservación no se desarrollen correctamente y sugirió restablecer la información en la página web, ya que la difusión es una de las principales líneas de acción para evitar la extinción de estas especies. ${ }^{56}$

Con los resultados a la vista, consideramos que el futuro de la recuperación y conservación del huemul en particular — pero aplicable a muchas otras especies amenazadas y en peligro de extinción con los debidos estudios preliminares- se encuentra en los centros de reproducción en cautiverio.

Para la utilización posible de estas tecnologías reproductivas habrá que tener presente que es necesario conocer en más profundidad la fisiología de la reproducción de las especies silvestres $y$, en este sentido, los programas de cría en cautividad están llamados a desempeñar un papel muy importante en la adquisición de este conocimiento. Dichas biotecnologías ya han demostrado su utilidad a la hora de recuperar especies que no podían salvarse por sí mismas de la extinción, ya sea porque habían alcanzado un número muy bajo de individuos, o porque la fragmentación de las poblaciones en núcleos pequeños y aislados había conducido a un aumento de la consanguinidad tal que impedía la reproducción «natural». ${ }^{57}$

Valga de ejemplo la Estación Experimental de Zonas Áridas (EEZA) establecida en España utiliza programa de cría en cautividad. Para ello se cuenta con la colaboración de numerosos núcleos zoológicos, por los que se dispersan pequeños grupos de individuos del Programa de Cría. Esto permite contar con una mayor disponibilidad de espacio y, por tanto, poder aumentar el tamańo de la población cautiva, pero, sobre todo, evitar que en caso de enfermedad o epidemia se vea afectada el conjunto de la población cautiva. Actualmente participa en proyectos de conservación de gacelas norteafricanas a través de programas de reintroducción y reforzamiento de gacela dorcas y gacela dama en Senegal. ${ }^{58}$

Al ser el país con mayor biodiversidad de Europa, este mecanismo también se ha aplicado en la acuicultura, promoviendo la cría en cautividad de las especies en peligro y su posterior reintroducción controlada en el medio natural.

Retomando la situación del huemul, aunque este es el único cérvido sudamericano en peligro de extinción según UICN, es la especie con menos información conocida. En contraste, los cérvidos solo considerados amenazado (venado de la pampa) o vulnerable (venado de los

56 Auditoría General de la Nación, "Informe de auditoría. Examen de la gestión del Programa de Conservación de Especies Amenazadas. Período auditado: del 01-01-2011 al 31-03-2016”, https://www.agn.gov.ar/files/informes/2018_062info.pdf

57 E. Roldán, J. Garde, "Biotecnología de la reproducción y conservación de especies en peligro de extinción”, en Los retos medioambientales del siglo XXI. La problemática...

58 Para conocer más acerca del Programa de cría en cautividad de la EEZA, visítese http://www.eeza.csic.es/es/programadecria.aspx 
pantanos) cuentan con un grado mucho más elevado de conocimiento: ambos cuentan con programas elaborados de centros de conservación. ${ }^{59}$

Las brechas de información existentes pueden acortarse mediante la investigación de huemul en semicautiverio, y además proveer de animales y/o genética para reintroducciones. Igualmente, y como corolario, vale la pena aclarar que las poblaciones cautivas solo son un apoyo para mejorar el conocimiento de base y lograr planteles saludables para reinserción, no son un sustituto de las poblaciones silvestres.

Considerando la ausencia de recuperación de las subpoblaciones de huemul en las últimas décadas, a pesar de las acciones llevadas a cabo, y una continua dilación en la aplicación de herramientas ex situ, se puede conceptualizar la flecha causal del tiempo de manera siguiente:

(i) Continuará la declinación numérica y extinciones locales;

(ii) Será incierto llegar a encontrar animales fundadores para centros; y

(iii) Quizás, lamentablemente, una probabilidad decreciente de lograr una recuperación de huemul. ${ }^{60}$

Por ello, se torna indispensable realizar un aggiornamiento de los postulados clásicos de nuestro sistema jurídico, para adaptarlos a la realidad, del derecho ambiental.

En este mismo sentido, hay que continuar impulsando las políticas de repoblación sistemática mediante las estrategias de conservación, restauración y manejo sostenible de las poblaciones salvajes y sus hábitats. Y de esta manera, utilizar la repoblación como medida de restauración temporal, realizándose a partir de líneas naturales obtenidas de las correspondientes poblaciones autóctonas o naturalizadas. ${ }^{61}$

\section{REFERENCIAS}

- Auditoría General de la Nación. "Informe de auditoría. Examen de la gestión del Programa de Conservación de Especies Amenazadas. Período auditado: del 01-01-2011 al 3103-2016”. https://www.agn.gov.ar/files/informes/2018_062info.pdf

59 E. Escobar Ruiz, J. Smith, W. Flueck, "El Huemul...

60 E. M. Escobar Ruiz, Z. Gizejewski, S., Hoby, W. T. Flueck, J. M. Smith-Flueck, "El valor de los centros de conservación para la supervivencia del huemul", en El Huemul de...

61 OESA - Fundación Biodiversidad, Caracterización de la cría... 
- Bonilla Maldonado, D., "Justicia ambiental, normas jurídicas y acción política”. En. Derecho ambiental y justicia social, comp. por G. Hardín, C. Stone, C. Rose. Estudio preliminar de Colin Crawford. Bogotá: Siglo del Hombre Editores, 2009.

- Escobar Ruiz, E.; J. Smith y W. Flueck. "El Huemul. Shoonem. Madera que se mueve/re". Fundación SHOONEM, 2018. https://deerlab.org/tmp/Escobar2018libroHuemul.pdf

- Escobar Ruiz, E. M; Z. Gizejewski; S. Hoby; W. T. Flueck; y J. M. Smith-Flueck. "El valor de los centros de conservación para la supervivencia del huemul". En El Huemul de Aysén y otros rincones, editado por Irarte, A., Donoso, D., Segura, B., Tirado, M. Chile: Ediciones Secretaría Regional Ministerial de Agricultura de la Región de Aysén y Flora y Fauna. https://www.researchgate.net/publication/319177637_EL_VALOR_DE_ LOS_CENTROS_DE_CONSERVACION_PARA_LA_SUPERVIVENCIA_DEL_ HUEMUL

- Espunyes Nozières, J. Reintroducción de especies amenazadas. Problemáticas y recomendaciones. España: Universitat Autònoma de Barcelona, 2011/2012. https://ddd. uab.cat/pub/trerecpro/2011/85780/reiespame.pdf

- López Alfonsín, M. Derecho ambiental. Buenos Aires: Astrea, 2012.

- Lorenzetti, R. La protección jurídica del ambiente. LL, 1997-E-1463.

- OESA - Fundación Biodiversidad. Caracterización de la cría en cautividad y repoblación de especies de interés a través de la acuicultura. Madrid, España: Fundación Biodiversidad, 2018. https://www.mapa.gob.es/app/jacumar/recursos_informacion/Documentos/ Publicaciones/338_Caracterizacion_cria_cautividad_repoblacion_especies_interes.pdf

- Paulich, N. "Increasing Private Conservation through Incentive Mechanisms". Stanford Journal of Animal Law \& Policy 3 (2010): 106-158. https://law.stanford.edu/wp-content/ uploads/2018/05/paulich.pdf

- Petrella, A. "La justicia ambiental a la luz del constitucionalismo social". LL, Buenos Aires, 2011-B-19.

- Povilitis, A. "El estado actual del huemul (Hippocamelus bisulcus) en Chile central". Gayana (Concepc.) [online], 66, n. ${ }^{\circ} 1$ (2002): 59-68.

https://scielo.conicyt.cl/scielo.php?script=sci_arttext\&pid=S0717-65382002000100008\#1 https://doi.org/10.4067/S0717-65382002000100008

- Povilitis, A.; D. Parsons; M. Robinson; y D. Becker. "The Bureaucratically Imperiled Mexican Wolf". Conservation Biology, Society for Conservation Biology 20, n. ${ }^{\circ} 4$, (2006): 942-945. 
https://www.researchgate.net/publication/6866374_The_Bureaucratically_Imperiled_ Mexican_Wolf

https://doi.org/10.1111/j.1523-1739.2006.00489.x

PMid:16922210

- Roldán, E.; J. Garde. "Biotecnología de la reproducción y conservación de especies en peligro de extinción". En Los retos medioambientales del siglo XXI. La problemática de la conservación de la biodiversidad en España, ed. por Montserrat Gomendio Kindelán, 307338. España: Fundación BBVA.

http://www.gebir.csic.es/descargas/Roldan_Garde_ReproTech_FBBVA.pdf

- Ruhl, J. B. "How to Kill Endangered Species, Legally: The Nuts and Bolts of Endangered Species Act 'HCP' Permits for Real Estate Development". The Environmental Lawyer, 5 (1998/1999): 345-405. https://www.researchgate.net/publication/228137717_How_ to_Kill_Endangered_Species_Legally_The_Nuts_and_Bolts_of_Endangered_Species_ Act_'HCP'_Permits_for_Real_Estate_Development

- UICN. "Categorías y Criterios de la Lista Roja de la UICN: Versión 3.1". Comisión de Supervivencia de Especies de la UICN. UICN, Gland, Suiza y Cambridge, Reino Unido, 2001. https://www.iucn.org/es/content/categor\%C3\%ADas-y-criterios-de-la-lista-rojade-la-uicn-versi\%C3\%B3n-31-segunda-edici\%C3\%B3n

RECIBIDO: $17 / 04 / 2019$

APROBADO: 2/05/2019 